\title{
BESSEL-LIKE BEAMS WITH Z-DEPENDENT CONE ANGLES
}

\author{
Vladimir N. Belyi ${ }^{1}$, Andrew Forbes ${ }^{2,3}$, Nikolai S. Kazak ${ }^{1}$, Nikolai A. Khilo ${ }^{1}$, Piotr I. Ropot ${ }^{1}$ \\ ${ }^{1}$ B.I. Stepanov Institute of Physics, NASB, 68, Nezavisimosti ave., 220072 Minsk, Belarus \\ ${ }^{2}$ CSIR National Laser Centre, PO Box 395, Pretoria 0001, South Africa \\ ${ }^{3}$ School of Physics, University of KwaZulu-Natal, Private Bag X54001, Durban 4000, South Africa
}

\begin{abstract}
ABSRACT
A new type of Bessel-like optical beams, which is distinguished by the dependence on the cone angle from the longitudinal coordinate, is investigated. Such beams have the properties of Bessel beams (ring-spatial spatial spectrum) as well as Gaussian beams (keeping the transverse profile at any distances). This new type of beams can be obtained in optical system composed of lens axicon doublet and conical lens. An experimental set-up for producing such beams is realized. It is shown that depending on its parameters the scheme allows one to produce z-dependent Bessel-like beams, whose spatial spectra change from Bessel function to shifted Gaussian one. It is establish theoretically and confirmed experimentally that on-axial intensity of z-dependent Bessel-like beam could be higher than that of incident Gaussian beam.
\end{abstract}

Key words: Bessel beams, axicon, Gaussian beam, diffraction integral, spatial spectrum.

\section{INTRODUCTION}

The zero-order Bessel beam $J_{0}$ as a mathematical construction was firstly introduced by Durnin [1]. Such beams can be produced by using annulus and a lens [2], a hologram [3], or an axicon [4,5]. Apart of passive methods for production of Bessel beams, the intracavity generation can be used [6-9].

The use of an axicon provides the most efficient method for producing Bessel beam, because the axicon has a higher transmittance than an annulus and produces no higher-order diffracted beams as there generated in a holographic axicon and axicon gratings. High efficient method for production of Bessel beam can be also based on using anisotropic crystals $[10,11]$.

The interest in Bessel light beams is connected, first of all, with the nondiffracting nature of these beams and with the effect of self-reconstruction of the transverse profile after shadowing (see, for example, [12]). These properties are the advantage of Bessel beams as compared with traditional Gaussian ones. Inside the nondiffracting region the Bessel light beam does not change its profile. But at the boundary of this region the beam abruptly transforms into a conical field with the characteristic ring-shaped intensity distribution ("double-face" effect). The significant difference of the near-field and far-field spatial structure can be considered as the disadvantage of such beams in contrast to Gaussiantype beams which preserve their profile while propagating in the free space over any distances.

The "double-face" effect can be partially weakened when going to Bessel beams with very small cone angle $\gamma$ as the non-diffraction beam length is inversely proportional to the angle $\gamma$. There is an elegant possibility to eliminate the "double-face" effect for Bessel beams. This includes the generation of beams with a decreased cone angle $\gamma$ during beam propagation. The beams with the decreasing cone angle $\gamma(\mathrm{z})$ are also more interesting in practice.

In so doing, if at $\mathrm{z} \rightarrow \infty$ the limiting value of angle $\gamma(\mathrm{z})$ at is equal to zero, such beams will hold an intermediate position between Bessel and Gaussian beams in respect to their properties. In what follows the optical beams formed in such schemes will be referred to as z- dependent Bessel-like beams. To realize such beams, use can be made of a modification of linear axicons and various lens systems with spherical aberration and anastigmatic lens axicons with the reflecting spherical surfaces [12-16]. Such schemes have been investigated before, mainly with the aim of obtaining the maximally uniform an-axis profile and constant diameter of the central spot size at the given focal segment as well as with the aim of minimization of astigmatism that is typically large for conical optics [17]. In the papers [18-19] the possibility is shown of achieving of a high transverse resolution at large distances in a scheme of a defocused Galileantype telescope with negative spherical aberration. However until now there has not been a detailed investigation of the transverse structure of z-dependent Bessel beams in the near and far zones of diffraction and the possibility of managing the axial intensity of a generated beam by the change of scheme parameters.

Laser Beam Shaping X, edited by Andrew Forbes, Todd E. Lizotte, Proc. of SPIE Vol. 7430, 74300E · (c) 2009 SPIE · CCC code: 0277-786X/09/\$18 - doi: 10.1117/12.824687 
In particular, of great practical interest is the study of the possibility of achieving the beam intensity higher than that in Gaussian beam which used in the scheme as an input one. This problem is rather comprehensive and can be investigated for all the proposed schemes intended for generation of z-dependent Bessel-like beams.

In this paper, a scheme is proposed and analyzed which includes aberration free optical elements consisting of an lens-axicon doublet and second axicon (Fig.1). It is shown that such a scheme allows one to produce z-Bessel beams that have a number of interesting properties, first of all in the far zone of radiation. Also the local spatial-angular spectra are investigated which can be obtained by limiting z-Bessel beams with an circular aperture. Main features of z-Bessel beams obtained by the theoretical analysis are compared with experimental results.

\section{OPTICAL SCHEME AND MECHANISM OF PRODUCTION OF BESSEL-LIKE BEAM WITH Z- DEPENDENT CONE ANGLE}

One of the possible optical schemes that allows one to obtain the Bessel beam with $\mathrm{z}$ - dependent cone angle $\gamma=$ $\gamma(\mathrm{z})$ is shown on the Fig. 1. The scheme consists of two axicons $A x_{1}$ and $A x_{2}$ and spherical lens $L$ with the focal length $F$. The axicons $A x_{1}$ and $A x_{2}$ are characterized with parameters $\gamma_{1}$ and $\gamma_{2}$ which are cone angles of Bessel beams generated by these axicons respectively.

The simplified analysis of the scheme can be made within the frame of geometrical optics. Incoming Gaussian beam is transformed with the spherical lens and axicon $A x_{1}$ into the ring field in the focal plane $F$. As it is seen from the scheme, the ring radius equals to $R_{1}=F \operatorname{tg}\left(\gamma_{1}\right)$. The ring width is known to be in inverse proportion to the diameter of the incoming Gaussian beam, and the angular divergence is directly proportional to the diameter. Thus, if an input Gaussian beam is well collimated, the second axicon $A x_{2}$ will be illuminated with a ring-like beam with a high divergence angle $\theta$. Besides, this annular beam belongs to the conical type with the average cone angle $\gamma_{1}$. The second axicon refracts the incoming field in the direction of optic axis. Thus, after passing through the axicon $A x_{2}$ (see Fig.1) this beam is transformed so that its carrier spatial frequency is decreased but the divergence angle is fixed. As a result,

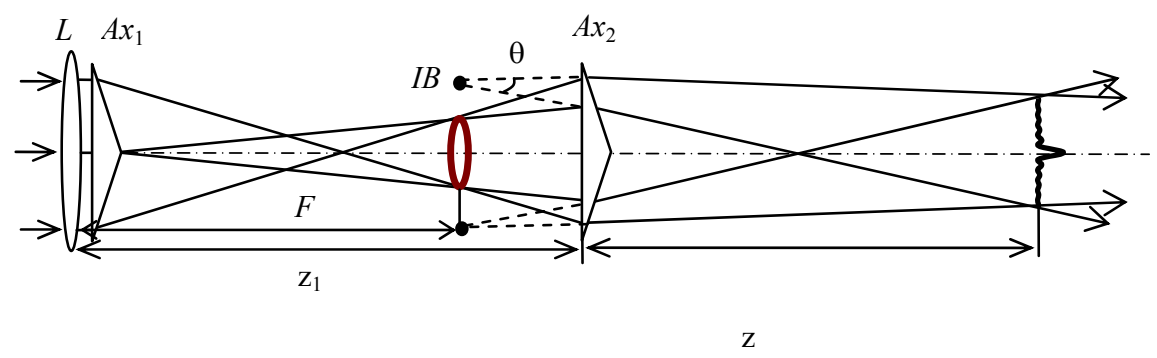

Figure 1. The optical scheme of formation of Bessel-like beam with the cone angle reducing along the direction of propagation.

the region behind the axicon $A x_{2}$ will be illuminated with imaginary ring-like light source (IB on Fig.1). This source can be also located after the axicon $A x_{2}$. That is in the case if an inequality $\mathrm{z}_{1}<F$ takes place.

As can be seen from Fig. 1, the crossing angle of a light rays on the optical axis will be reduced with the distance $z$. But the crossing angle remains the same at any distances $\rho$ from the optical axis at fixed longitudinal coordinate $z$. Under fulfillment of the approximate equality $\gamma_{1}+\theta \approx \gamma_{2}$, the maximal crossing angle converges to zero at $\mathrm{z}$.

Taking into account the cylindrical symmetry of optical scheme, it can be concluded from the above analysis that the output field is the Bessel - like optical beam with $\mathrm{z}$ - dependent cone angle.

\section{ANALYTICAL CALCULATION OF THE DIFFRACTION INTEGRAL AND NUMERICAL ANALYSIS}

To determine the output field in the scheme of Fig.1, a two-stage calculation of the Fresnel diffraction integral has been performed. At the first stage the field at the input plane of the second axicon is calculated:

$a_{1}\left(\rho, z_{1}\right)=-\frac{i}{\lambda z_{1}} \exp \left(\frac{i k_{0} \rho^{2}}{2 z_{1}}\right) \int_{0}^{R_{a 1}} \exp \left(-\frac{\rho_{1}^{2}}{\rho_{0}^{2}}-i k_{0} \gamma_{1} \rho_{1}-\frac{i k_{0} \rho \rho_{1}}{z_{1}} \cos \left(\varphi-\varphi_{1}\right)\right) \rho_{1} d \rho_{1} d \varphi_{1}$ 
where $\frac{1}{\rho_{0}^{2}}=\frac{1}{w_{0}^{2}}+\frac{i k_{0}}{2 F}-\frac{i k_{0}}{2 z_{1}}, R_{\mathrm{a} 1}-$ is the radius of the first axicon, $w_{0}-$ the halfwidth of input Gaussian beam. The calculation of integral, using the stationary phase method, gives

$a_{1}\left(\rho, z_{1}\right)=-\frac{i F}{z_{1}-F} \sqrt{1-\frac{\gamma_{1} z_{1}}{\rho}} \exp \left[\frac{i k_{0}}{2 z_{1}}\left(\rho^{2}+\frac{z_{1} / F-1+i z_{1} / z_{0}}{\left(z_{1} / F-1\right)^{2}+\left(z_{1} / z_{0}\right)^{2}}\left(\rho-\gamma_{1} z_{1}\right)^{2}\right)\right]$,

where $z_{0}=k_{0} w_{0}^{2} / 2$

As it is seen from Eq. (2) the field incident on the second axicon appears to be the shifted Gaussian beam. The wavefront curvature is positive at $\mathrm{z}_{1}>F$ and negative in the opposite case $\mathrm{z}_{1}<F$.
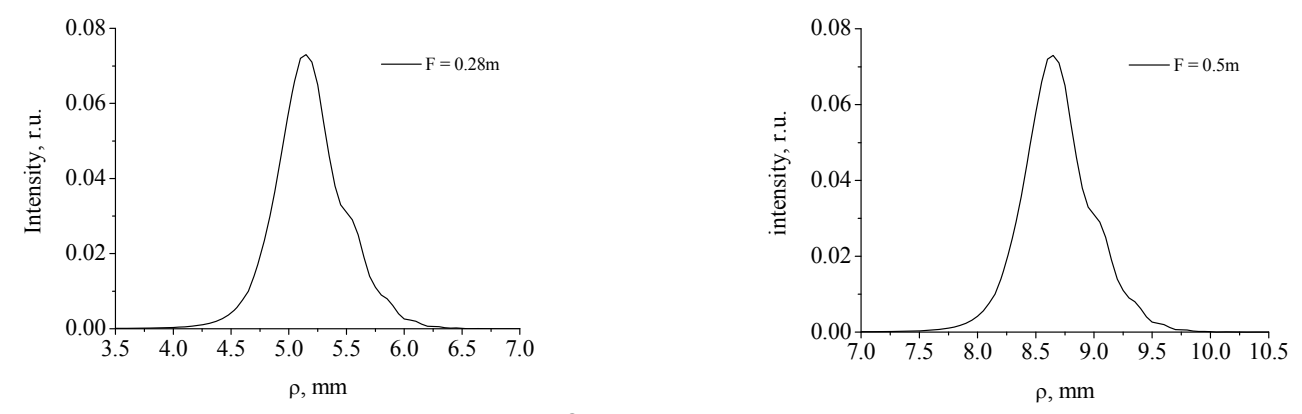

a

Figure 2 Transverse intensity distribution at the entrance plane of axicon 2. focal length $F=0.28 \mathrm{~m}$ (a); $0.5 \mathrm{~m}$ (b)

Numerical calculation of diffraction integral (1) was performed and compared with analytical results. From Fig. 2 it is seen that field incident on second axicon is close to shifted Gaussian beam. Some difference caused by the diffraction at sharp tip of first axicon.

Further the Eq. (1) is used while calculating the second diffraction integral:

$a(\rho, z)=-\frac{i}{\lambda z} \int_{0}^{R_{a 2}} a_{1}\left(\rho_{1}, z_{1}\right) \exp \left(\frac{i k_{0}\left(\rho^{2}+\rho_{1}^{2}-2 \rho \rho_{1} \cos \left(\varphi-\varphi_{1}\right)\right)}{2 z}-i k_{0} \gamma_{2} \rho_{1}\right) \rho_{1} d \rho_{1} d \varphi_{1}$

On calculating, the following equation for complex amplitude of the output field $a\left(\rho, z_{1}, z\right)$ is obtained:

$a\left(\rho, z_{1}, z\right)=\exp \left(\frac{i k_{0} \rho^{2}}{2 R_{1}\left(z_{1}, z\right)}\right)\left[f_{+} \exp \left(-i k_{0} \gamma\left(z_{1}, z\right) \rho\right)-i f_{-} \exp \left(i k_{0} \gamma\left(z_{1}, z\right) \rho\right)\right]$

where $f_{ \pm}\left(\rho, z_{1}, z\right)=\frac{F}{2\left(z+z_{1}-F\right)} \sqrt{\left[\gamma_{2}-\gamma_{1}\left(1+\frac{z_{1}}{z}\right)\right] \frac{z}{\rho} \pm 1}$,

$\frac{1}{R_{1}\left(z_{1}, z\right)}=\frac{1}{z}+\frac{1}{\bar{R}\left(z_{1}, z\right)}, \bar{R}\left(z_{1}, z\right)=z\left(1+\frac{z}{z_{1}}+\frac{z}{R\left(z_{1}\right)}\right), R\left(z_{1}\right)=z_{1}\left(\frac{z_{1}}{F}-1\right)$

$\gamma\left(z_{1}, z\right)=\frac{z}{\bar{R}\left(z_{1}, z\right)}\left(\gamma_{2}+\frac{\gamma_{1} F}{z_{1}-F}\right)$

Using the known asymptotical form for the zeroth order Bessel function of the first kind $J_{0}(z) \approx \sqrt{2 / \pi z} \cos (z-\pi / 4)$ we arrive at 
$a\left(\rho, z_{1}, z\right) \approx f\left(\rho, z_{1}, z\right) \exp \left(\frac{i k_{0} \rho^{2}}{2 R_{1}\left(z_{1}, z\right)}-\frac{i k_{0} \gamma\left(z_{1}, z\right)^{2} \bar{R}\left(z_{1}, z\right)}{2}\right) J_{0}\left[k_{0} \gamma\left(z_{1}, z\right) \rho\right]$

where $f\left(\rho, z_{1}, z\right)=\frac{1}{2} \sqrt{\frac{\pi k_{0} \gamma\left(z_{1}, z\right) \rho}{2}}\left(f_{+}\left(\rho, z_{1}, z\right)+f_{-}\left(\rho, z_{1}, z\right)\right)$.

As is shown from the numerical calculation, the amplitude function $f\left(\rho, z_{1}, z\right)$ in Eq. (8) has only a weak dependence on the transverse coordinate $\rho$. Therefore, the Eq. (8) describes the field with Bessel transverse intensity distribution. The dependence of the amplitude function $f\left(\rho, z_{1}, z\right)$ on the longitudinal coordinate $\mathrm{z}$ is essential here and will be investigated below. Quadratic-phase multiplier in Eq. (8) describes the wavefront curvature of the beam.

The dependence of cone angle $\gamma$ on the longitudinal coordinates $z_{1}$ and $\mathrm{z}$ can be represented in the form

$\gamma\left(z_{1}, z\right)=\frac{\gamma_{2} z_{1}+\left(\gamma_{1}-\gamma_{2}\right) F}{z_{1}+z-F}$

As is seen from Eq. (9), the cone angle always decreases when increasing z.

\section{NUMERICAL CALCULATION OF TRANSVERSE STRUCTURE OF Z-DEPENDENT BESSEL- LIKE BEAMS}

The numerical analysis of the diffraction integral (3) has been made. The following set of parameters has been used: wavelength $\lambda=0.63 \mathrm{mkm}$; cone angles $\gamma_{1}=0.5 \mathrm{deg}$.; $\gamma_{2}=0.92 \mathrm{deg}$.; half-width $w_{0}=1 \mathrm{~mm}$; length $z_{1}=1.9 \mathrm{~F}$. The focal distance $F$ was varied.

In the Fig. 3 the dependence of the axial beam intensity on the distance to the second axicon is shown. As is seen, it is a one-picked curve typical for Bessel beams. But the intensity oscillation here is rather weaker than in the scheme with one axicon. Further, it follows from the comparison of Fig. $3 \mathrm{a}$ and $3 \mathrm{~b}$ that a decrease in the focal length $\mathrm{F}$ leads to an increase of the region with a high intensity or the focal beam length.

When $\mathrm{z}$ increases up to several meters and more, there occurs a slow monotonous, decrease in the intensity, which is typical for Gaussian beams.

The study of the transverse intensity distribution, as well as of the spatial (angular) spatial spectrum of the generated beam, is of a great interest. For calculating the spatial spectrum, the known lens scheme (the focal length of the lens is $0,5 \mathrm{~m}$ ) was used.

It follows from the graphs comparison that the profiles of the intensity distribution of the field and spatial spectrum coincide here. This points to the fact that at this distance the far zone of the formed beam is realized. The novelty here is that the field in the far zone is not the annular one, which is typical for usual schemes with axicon. The oscillating beam, which has the intensity distribution similar to the Bessel one, is observed instead of an annular field.
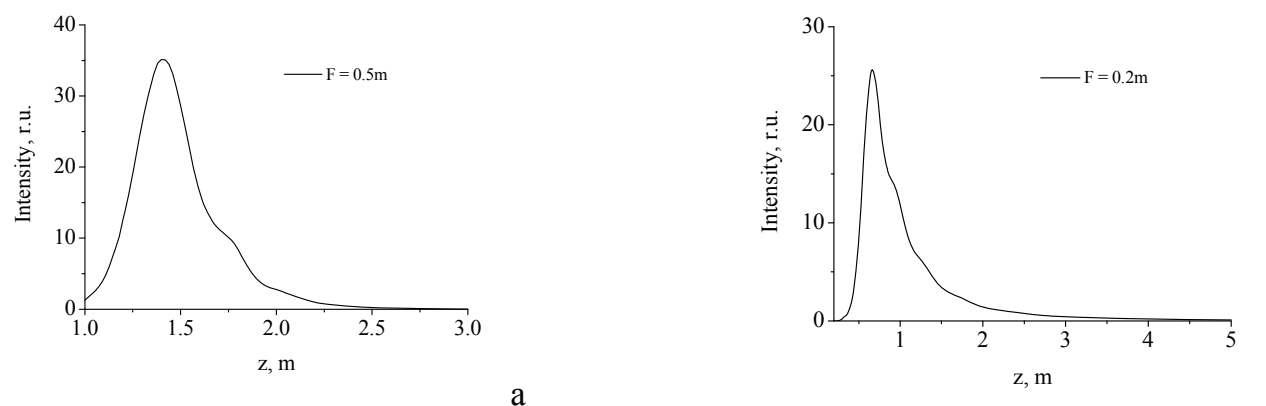

a

\section{b}

Figure 3. Dependence of the intensity on the beam axis from the transverse coordinate $\mathrm{z}$. Focal length $\mathrm{F}=0.5 \mathrm{~m}(\mathrm{a})$; $0.2 \mathrm{~m}(\mathrm{~b})$ 
In Fig. 4 are presented the dependences of the field and spatial spectrum intensity for a scheme containing a lens with a relative short-focal length $(\mathrm{F}=18 \mathrm{~cm})$.
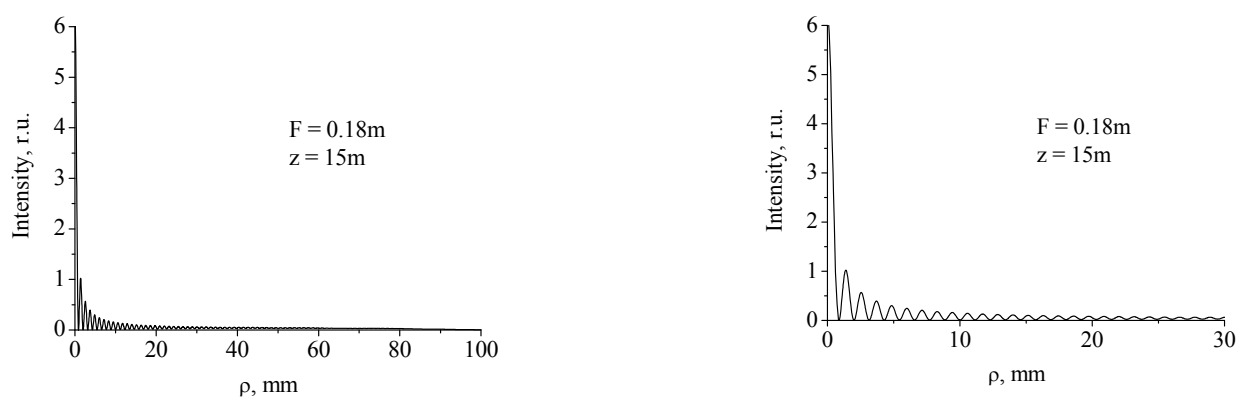

a

b
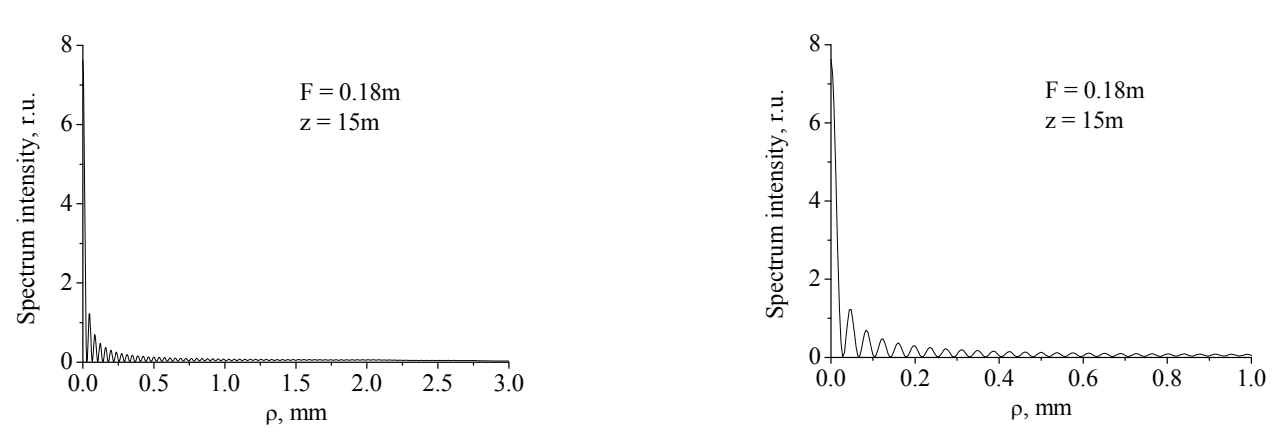

c

Figure 4. Calculated intensity distribution $(a, b)$ and spatial spectrum intensity $(c, d)$ in the scheme with the shortfocal length lens at the distance $\mathrm{z}=15 \mathrm{~m}$. The full-range graphs are $\mathrm{a}, \mathrm{c}$; their near-axial parts are $\mathrm{b}, \mathrm{d}$.

When $\mathrm{z}$ increases, the spatial spectrum shape is retained, whereas the oscillation frequency in the intensity distribution diminishes (Fig. 5).
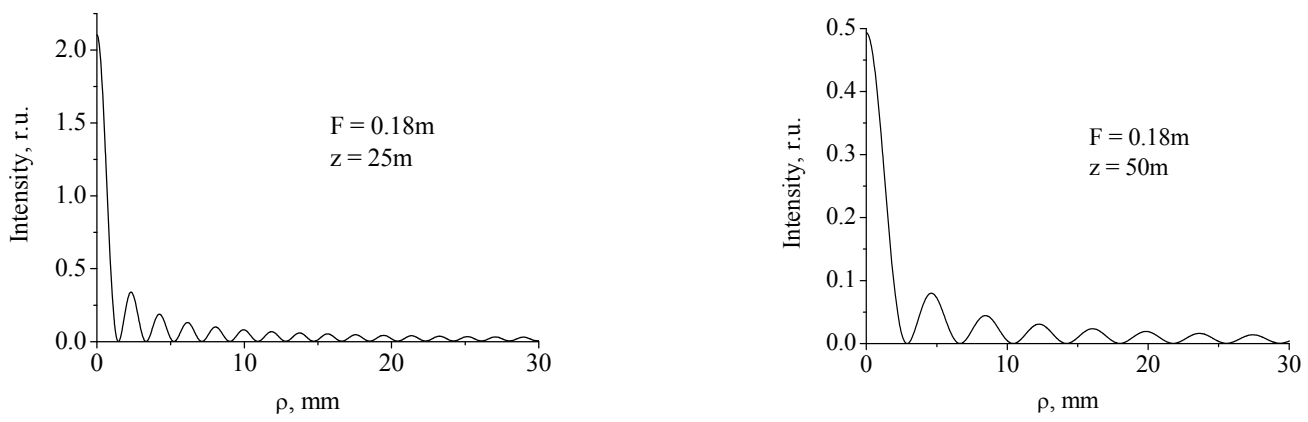

a

Figure 5. Variation of the frequency of intensity oscillations when the propagation length increases. $z=25 \mathrm{~m}$ (a) and $50 \mathrm{~m}(\mathrm{~b})$.

This confirms the above analytical result (Eq. (8)) on the formation in this scheme of the local Bessel beam, whose cone angle decreases with distance. At that the absence of the annular spatial spectrum, which is typical for Bessel beams, can be explained by a strong quadratic phase modulation of a beam (Eq. (8)). In particularly, it follows from Eqs. (6), (7) that the role of phase modulation becomes greater, when the F decreases, which is related to a decrease in the radius of curvature of the wave front.

When the focal length F increases, the field and spectra structures change essentially (Fig. 6). It follows from Fig.6 that as the focal distance increases, the far field structure changes smoothly from Bessel to annular one. The annular component of the total field is characterized here by a large width, which is the result of a relatively high divergence of the annular field incident upon the second axicon. 
Here in the near-axial region the quasi-Bessel oscillations are retained for all time, and their intensity decreases while increasing the focal length F. Here and further, in accordance with existing literature (see, for example, [20]), the term "quasi-Bessel beam" denotes some approximation to idealized $J_{0}$-beam.

Note, that in these calculations the distance between axicons was taken as $1.9 \mathrm{~F}$. That is why when the focal distance $\mathrm{F}$ increases, the divergence of the beam incident upon the second axicon decreases. This leads to the domination of the annular component in the spatial spectrum of the output beam.
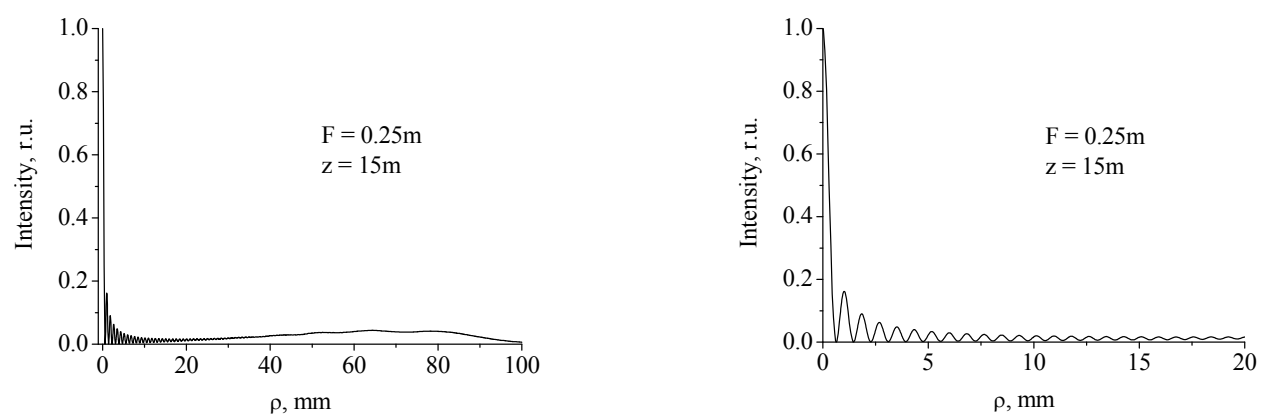

a
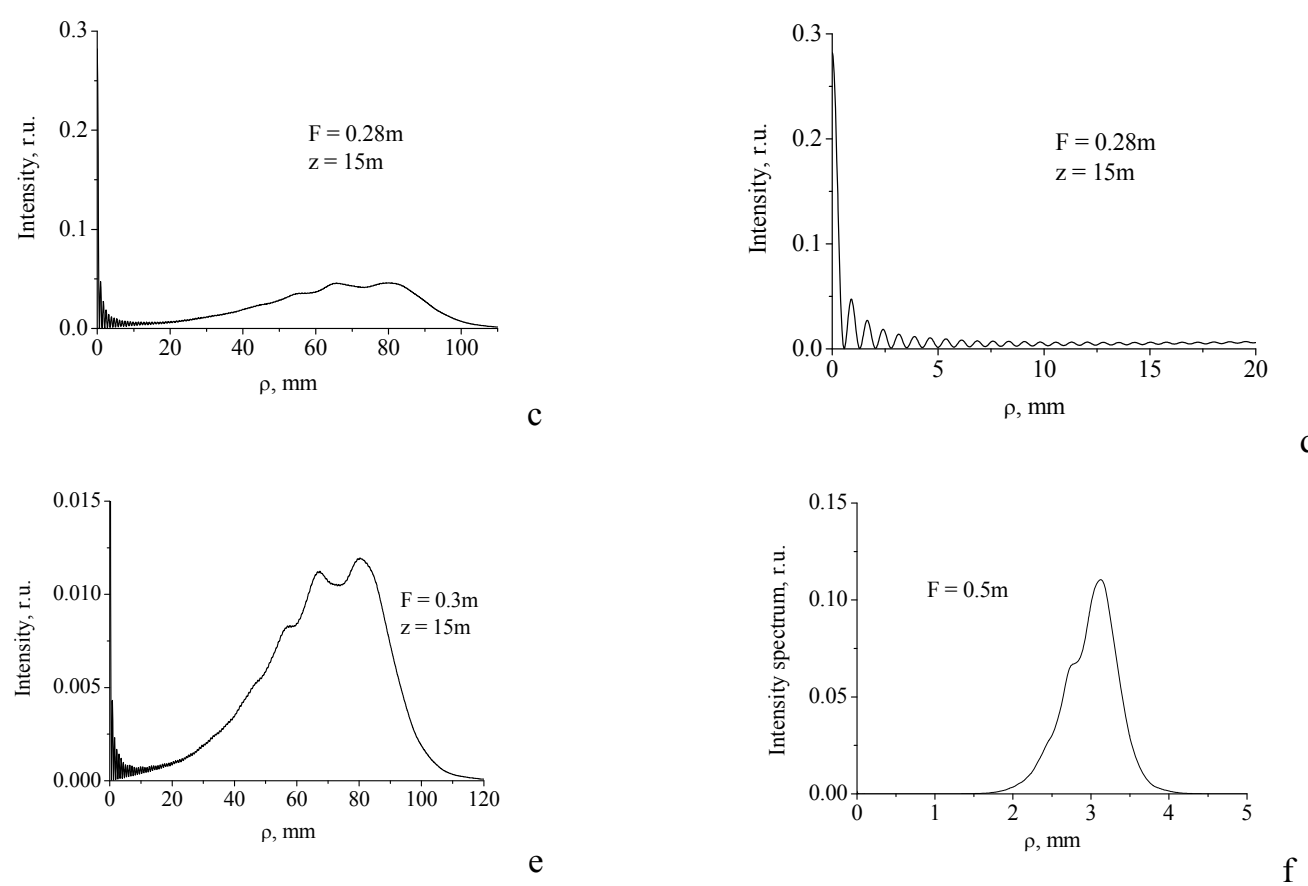

Figure 6. Dependence of the field intensity distribution in the far zone on the change of the focal length of lens. $F=$ $25 \mathrm{~cm}(\mathrm{a}, \mathrm{b}) ; 28 \mathrm{~cm}(\mathrm{c}, \mathrm{d}) ; 30 \mathrm{~cm}(\mathrm{e})$. also shown the spatial spectrum intensity at $\mathrm{F}=50 \mathrm{~cm}(\mathrm{f})$.

It should be pointed out that the behavior of the far zone profile shown in Fig.6 is similar to the known scenario of changing the intensity in the scheme with a single axicon in the transition region between Bessel and conical beams.

Thus, when the focal length $\mathrm{F}$ and correspondingly the distance $z_{1}$ in the scheme in Fig. 1 increases, the far-field generated by this scheme changes from the quasi-Bessel beam to an annular field.

\section{LOCAL SPATIAL SPECTRA OF Z-DEPENDENT BESSEL-LIKE BEAMS}

Also of a great interest is the investigation of local spatial spectrum of z-dependent Bessel-like beams, which are obtained when using the limiting circular apertures. It should be noted that when measuring at large distances, the aperture limitation is practically inevitable as a result of the increasing beam diameter. 

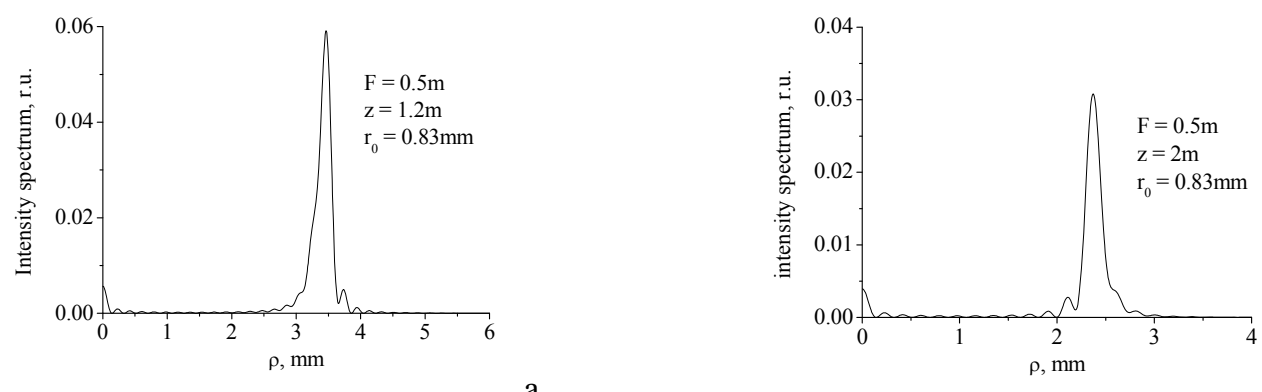

Figure 7. Local spectra of $z$-Bessel beams obtained in the scheme with $F=0,5 \mathrm{~m}$ at the distances $\mathrm{z}=1.2 \mathrm{~m}$ (a) and $\mathrm{z}$ $=2 \mathrm{~m}(\mathrm{~b})$ with the annular diaphragm with the radius of $0.83 \mathrm{~mm}$.

In Fig. 7 is given an example of computing the scheme with $\mathrm{F}=0,5 \mathrm{~m}$ (annular far zone) at two different distances $z$. When comparing with Fig. $6 f$, it is seen that the transverse beam limitation leads to the narrowing of the annular spatial spectrum. This phenomenon, at first sight, contradicts the wave theory, according to which the beam limitation causes the spatial spectrum broadening. However there is no contradiction here, because the narrowing of the annular region of spatial spectrum is accompanied by the appearing of the axial component, which can be explained as the broadening of the total spatial spectrum of the beam. Moreover, while removing the limiting aperture (increasing $\mathrm{z}$ ) there takes place a shift of local spatial spectrum towards the region of low frequencies (Fig. 7b), which is indicative of the decrease in the efficient cone angle. It should be pointed out that the local spectra in Fig. 7 are obtained in the near diffraction zone where the light intensity distribution is the quasi-Bessel one.

A similar calculation has been made for small values of $\mathrm{F}$ where the field of the far zone is the quasi-Bessel one (Fig. 8).
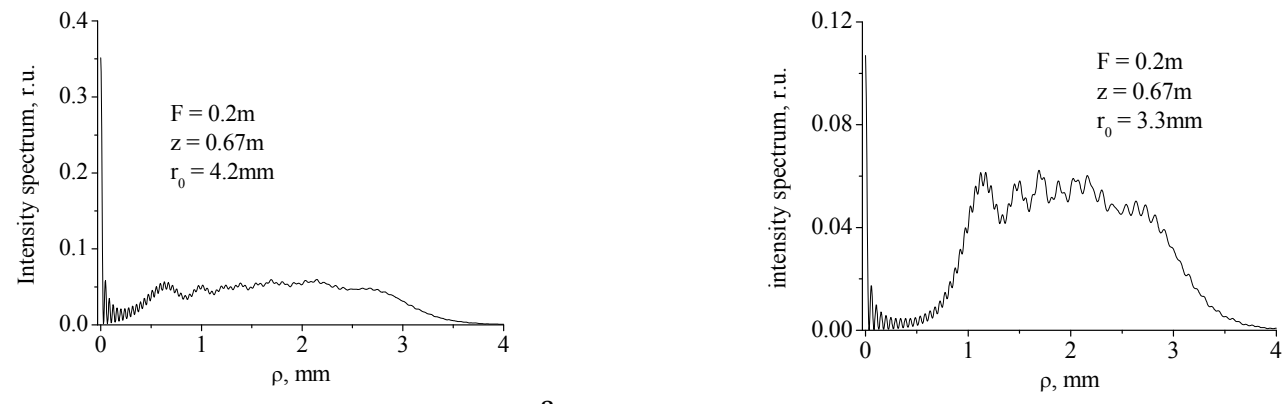

b

Figure 8. Local spectra of quasi-Bessel beams obtained in the scheme with $\mathrm{F}=0.2 \mathrm{~m}$ at the distance $\mathrm{z}=0.67 \mathrm{~m}$ with the use of annular diaphragm with the radius of $4.2 \mathrm{~mm}$ (a) and $3.3 \mathrm{~mm}(\mathrm{~b})$.

In this case, as the diaphragm established in the near beam zone is narrowed down, the enhancement of the annular component of spatial spectrum is observed. Therefore, the conical field is mainly concentrated in the central region of such beams.

Also the influence of diaphragm placed in the far zone of beam on its spatial spectrum has been investigated for schemes with the quasi-Bessel total spatial spectrum. It is shown that in this case the selection of some zone of the beam by the annular diaphragm leads to the filtration of the analogical zone in the spatial spectrum. This allows one to synthesize quasi-Bessel beams with a different spatial-angular structure due to the use of the corresponding spatial filters.

\section{ON-AXIAL INTENSITY OF Z-DEPENDENT BESSEL-LIKE BEAMS IN FAR-FIELD REGION}

The influence is studied of the ratio of the cone angles of axicons 1 and 2 (Fig. 1) on the intensity distribution in focal region of z-Bessel beam. In the Fig. 9 the intensity dependences on the beam axis from the longitudinal distance are depicted. For the comparison there we have used the input Gaussian beam in the scheme in Fig. 1 on the condition of its further free propagation. 

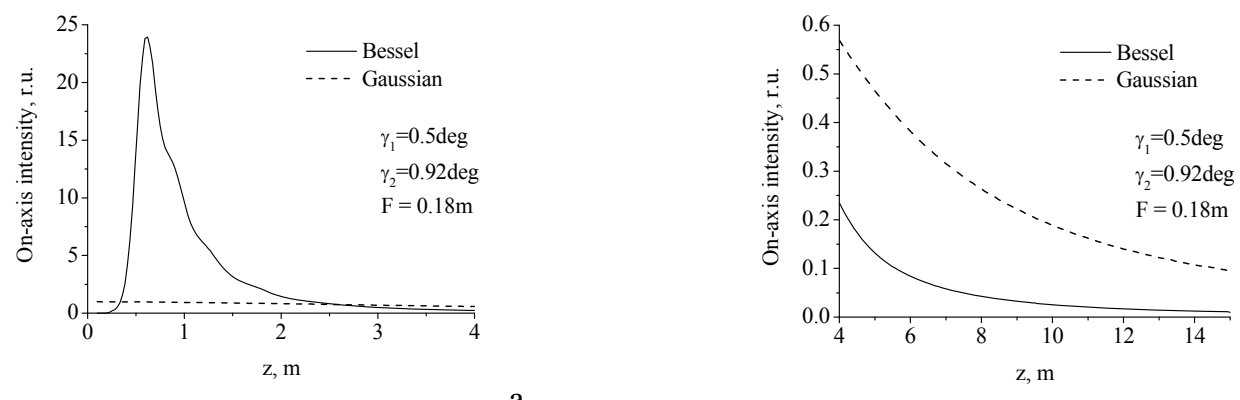

a

b
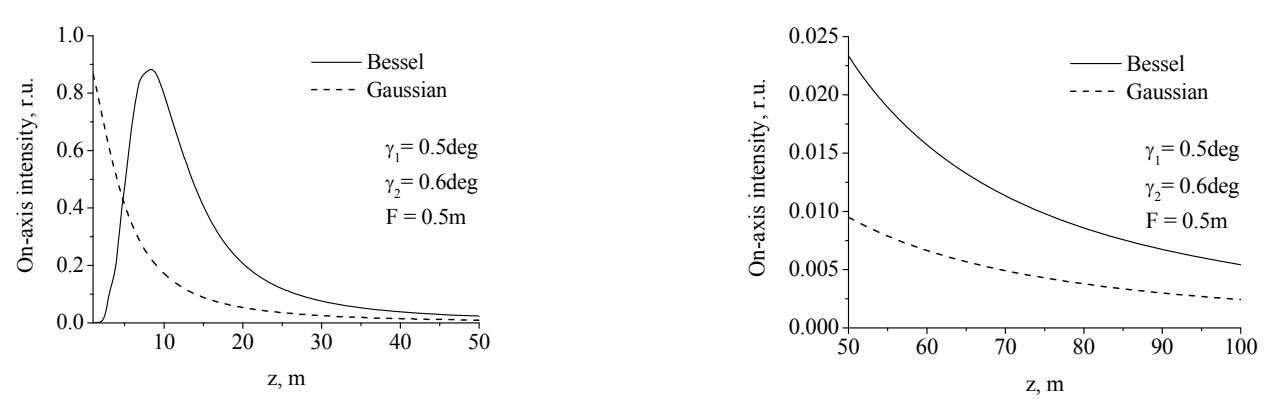

c

Figure 9. Axial intensity dependence of z-Bessel beam and the equivalent Gaussian beams in the scheme in Fig. 1.

It is seen in Fig. 9 that the intensity of the z-Bessel beam near the axicon is rather higher than the intensity of the Gaussian one. Such a behavior is known to take place for the usual scheme with one axicon as well. But far from axicon the intensity of z-dependent Bessel-like beam may be both smaller (Fig. 9a, b) and larger (Fig. 9c, d) than the intensity of Gaussian one. This fact differs considerably this scheme (Fig.1) from the traditional scheme with single axicon. At that the intensity of the z-Bessel beam turns out to be higher if the difference of the cone angles decreases (Fig. 9 c, d).

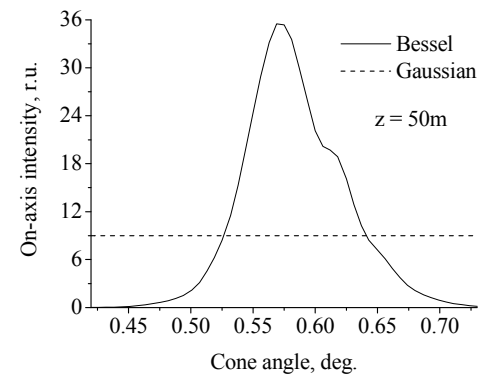

a

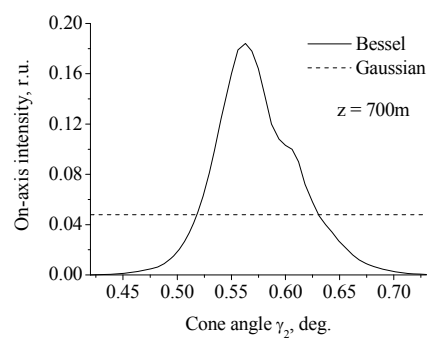

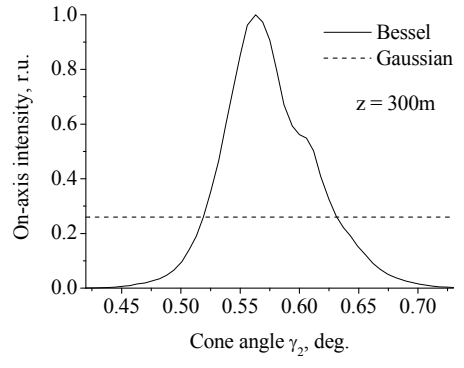

b

Figure 10. The dependence of axial intensity of $z$ Bessel and Gaussian beams at different distances $\mathrm{z}$ from the second axicon: $\mathrm{z}=50 \mathrm{~m}(\mathrm{a}) ; 300 \mathrm{~m}(\mathrm{~b}) ; 700 \mathrm{~m}(\mathrm{c}) . \mathrm{F}=$ $0.5 \mathrm{~m}$.

A special investigation has been made of the axial intensity at a given distance $\mathrm{z}$, depending on the ratio of the cone angles of two axicons in scheme Fig. 1. In Fig. 10 such dependences on the cone angle $\gamma_{2}$ are shown at the given angle $\gamma_{1}=0.5 \mathrm{deg}$. It is seen that there is the limited range of angles $\gamma_{2}$ within which the axial intensity of z-Bessel beam is higher. This range is located near the angle $\gamma_{1}$ in the region of large angles $\left(\gamma_{2}>\gamma_{1}\right)$. At that, for example, the Gaussian 
beam intensity at the curve maximum in Fig. 10 amounts approximately to 26 per cent from the intensity of z-Bessel beam.

Of importance is the problem of influence of the diameter of the input Gaussian beam on the ratio of axial intensities in the far zone. It should be pointed out that the above results have been obtained at $w_{0}=1 \mathrm{~mm}$ (Fig. 11).

From Fig. 11 it is seen that at $\mathrm{z}=50 \mathrm{~m}$ the far zone is realized. Here the field with high accuracy is described by the Bessel beam (the comparison showed that for Fig. $11 \mathrm{c}$ the cone angle of this beam equals to $1.6 \mathrm{deg}$.). At a distance of $15 \mathrm{~m}$ (Fig.11a) the field does not coincide with its spatial spectrum and has the pedestal that is typical for the transition zone from Bessel to conical field. The equality $\mathrm{z}_{1}=\mathrm{F}$ provides the small size of the annular beam on the second axicon (ring width is $1 \mathrm{~mm}$ from $13.5 \mathrm{~mm}$ up to $14.5 \mathrm{~mm}$ ). The comparison with Fig. 2 shows that the increase of half-width $w_{0}$ leads to the increase of the radius of the annular field, at a high axial intensity of the formed beam is achieved. Thus, at further increasing $w_{0}$, instead of whole axicon 2 , it is desirable to use only the annular fragment of axicon. At that the change of the scheme in Fig. 1 is possible with replacing refraction the axicon by a reflected conical mirror.

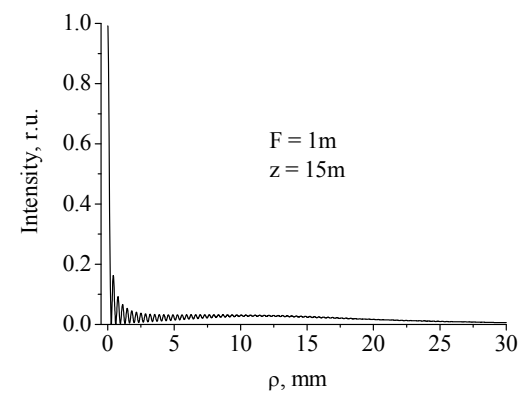

a
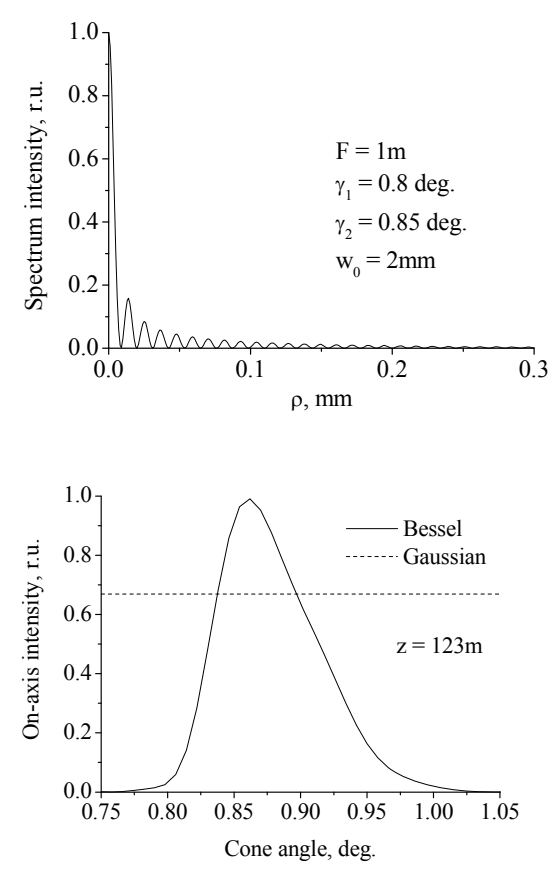

c$$
\text { e }
$$

Thus, for achieving the inequality $I_{\mathrm{Bs}}(\rho=0)>I_{\mathrm{Gs}}(\rho=0)$, the second axicon is necessary to have a little larger cone angle. In this case the field of the formed beam in the far zone is the quasi-Bessel one, and a relatively high intensity of the axial maxima is explained by slowing down its diffraction divergence due to the presence of side maxima of the beam. Unlikely the usual Bessel beam, in this case the property of the partial suppression of diffraction in the axial region becomes apparent at any distance. Note, that for providing high axial intensity of a beam reflecting axicons can also be used.
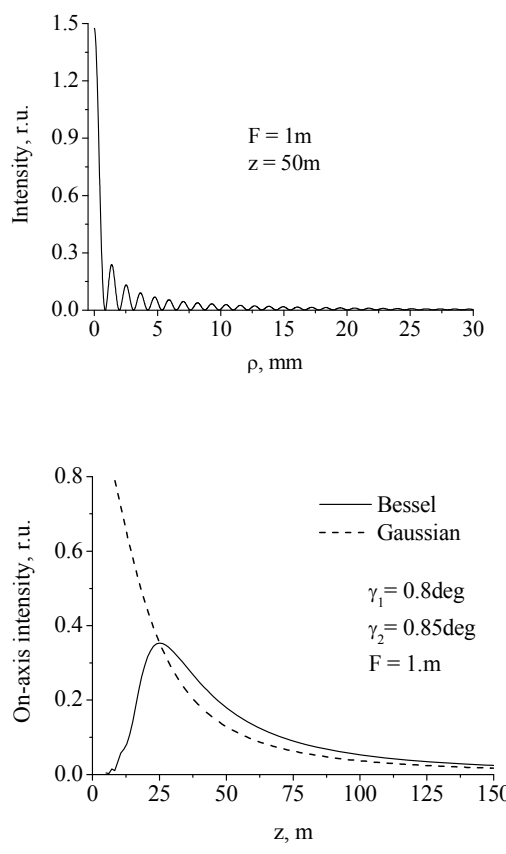

Figure 11. Main properties of the field at the radius of the input Gaussian beam of $2 \mathrm{~mm}$. The intensity distribution at $\mathrm{z}=15 \mathrm{~m}$ (a) and $\mathrm{z}=50 \mathrm{~m}$ (b); Fourierspectrum (c); on-axis intensity distribution (d); angular dependence of the axial intensity (e). Parameters: $\mathrm{F}=$ $1 \mathrm{~m} ; \gamma_{1}=0.8 \mathrm{deg}, \gamma_{2}=0.85 \mathrm{deg}$ (for Fig.11a-d); distance $\mathrm{z}_{1}=\mathrm{F}$. 


\section{EXPERIMENTAL RESULTS}

The scheme shown in Fig. 1 is experimentally tested, its parameters being: the cone angles of two axicons are $\gamma_{1}$ $=0.89$ and $\gamma_{2}=0.98$ deg. respectively, focal distance is $\mathrm{F}=0.5 \mathrm{~m}$, distance between two axicons is $\mathrm{z}_{1}=0.75 \mathrm{~m}$. An input Gaussian beam from a He-Ne laser after the preliminary spatial filtration has the halfwidth $w_{0}=2.5 \mathrm{~mm}$ (see Eq. (1)). The spatial spectra were formed by a spherical lens having a focal length of $0.5 \mathrm{~m}$. Images of output beams were captured with CCD-camera.

The evolution of the spatial beam profile with distance form axicon 2 is shown in Fig. 12. It is seen that the beam as a whole contains axial and annular components similar to the calculation graphs. Here, when the distance increases, the contribution of the annular component diminishes. And the field as a whole becomes similar to zdependent Bessel-like beam in its structure. In this case in the central region of the beam there exists the Bessel field (Fig. $12 \mathrm{~d}$, e, f), whose cone angle decreases with distance (as the period of the interference structure increases). Note that some azimuthal modulation of the beam intensity in Fig. 12 is conditioned by astigmatism of the optical system containing two axicons.
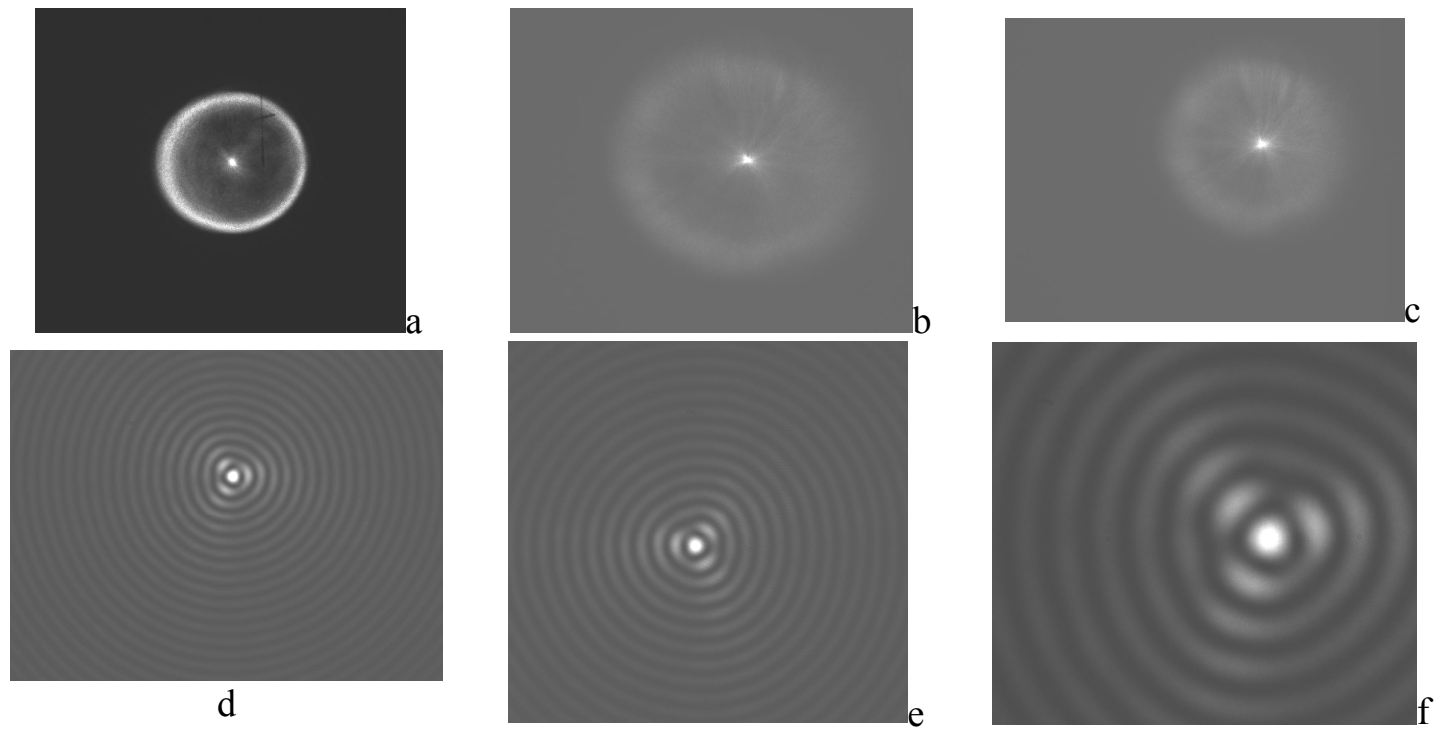

Figure 12. Experimentally measured transverse intensity distribution of the beam as a whole at different distances from the axicon $2: \mathrm{z}=0.9 \mathrm{~m}(\mathrm{a}) ; 5.2 \mathrm{~m}(\mathrm{~b}) ; 6.4 \mathrm{~m}(\mathrm{c})$ and the central part of the beam at the distances $\mathrm{z}=6.4 \mathrm{~m}(\mathrm{~d})$; $8.6 \mathrm{~m}(\mathrm{e}) ; 25 \mathrm{~m}(\mathrm{f})$

The spatial Furrier- spectrum of the beam as a whole is shown in Fig. 13. It is described with high accuracy by Bessel function.

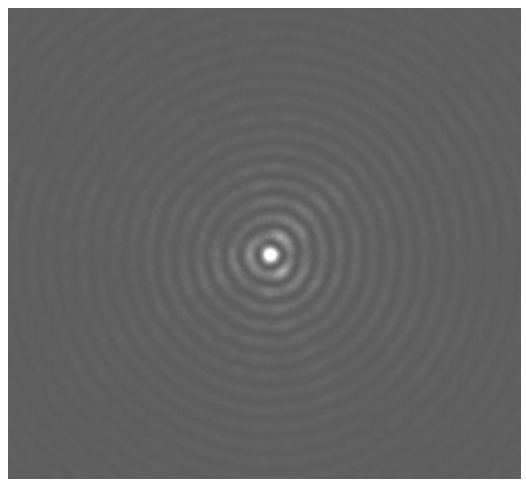

Figure 13. Experimentally measured spatial spectrum of the whole beam formed by the scheme in Fig. 1. 
Also the spatial Fourier-spectra are experimentally obtained of the beam local regions limited by the circular diaphragm (Fig.13). Unlike the spatial spectrum of the total beam (Fig.12), local spectra are annular ones. These findings lend support to the view that the central region of z-dependent Bessel-like beams, with a high degree of accuracy, is a usual $\mathrm{J}_{0}-$ Bessel beam.
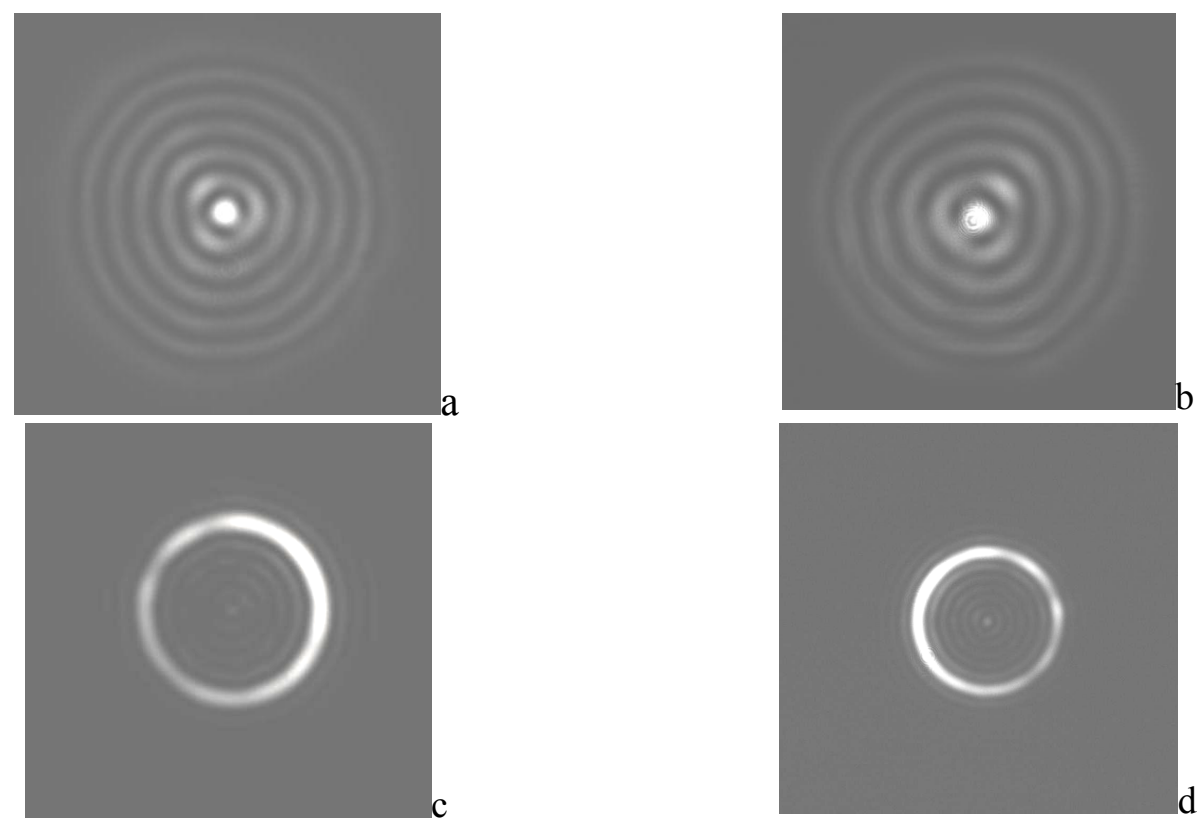

Figure 14. Images of near-axial regions of $\mathrm{z}$ - dependent Bessel-like beam taken at the distances $\mathrm{z}=6.2 \mathrm{~m}$ (a) and $9.2 \mathrm{~m}$ (b). Images of near-axial regions obtained at the beam limitation by the circular diaphragm and their spatial spectra (c,d) respectively. The diaphragm diameter is $d=2,9 \mathrm{~mm}$ in the case (a) and $\mathrm{d}=2.6 \mathrm{~mm}$ in the case (b).

The comparison of axial intensities of the z-dependent Bessel-like beam and input Gaussian beam has been made at a distance of $25 \mathrm{~m}$ from axicon 2 . The measurement has shown that the axial intensity of Gaussian beam is $\approx 2.5$ times smaller, which approximately corresponds to the calculated value that is equal to 2.7 . The difference may be caused, first of all, by inaccuracy of measurement of the cone angle of the axicons.

\section{CONCLUSION}

In conclusion, in this paper a study is made of the spatial-angular structure of z-dependent Bessel-like light beams formed by the system containing lens-axicon doublet and second conical lens. It is shown that in the far field such a scheme generates Bessel-like beams, whose cone angle depends on the longitudinal coordinate. Here the change of geometrical parameters of the scheme influences essentially the field in the far zone, which can extended from quasiBessel field to annular one. Therefore the generated light beams belong to the intermediate type ones spatial spectrum of which includes both axial and annular components.

It is shown that the spatial truncation of beams can also considerably influence their spatial spectrum, which provides a possibility of managing the spatial-angular structure of the formed light beams. It has been found that the beam axial intensity can be adjusted by changing the ratio of the cone angles of two axicons. At that in the case of the almost coincided cone angles the z-Bessel beam can have considerably higher axial intensity than the incident Gaussian beam. Thus, the investigation shows that z-Bessel beams posses a rich number of properties and can be of practical interest in the area of laser diagnostics, medicine, for example, for creation of more universal laser tweezers [21], and also in optical location [19].

\section{REFERENCES}

[1] J. Durnin, "Exact solutions for nondiffracting beams. I. The scalar theory," J. Opt. Soc. Am. A 4, 651-654 (1987).

[2] J. Durnin, J. J. Miceli Jr. and J. H. Eberly, "Diffraction free beams," Phys. Rev. Lett. 58, 1499 - 1501 (1987). 
[3] A. Vasara, J. Turunen, and A. T. Friberg, "Realization of general nondiffracting beams with computer-generated holograms," J. Opt. Soc. Am. A 6, 1748-1754 (1989).

[4] R.M. Herman, T.A. Wiggins, "Production and uses of diffractionless beams," J. Opt. Soc. Am. A 8, 932-942 (1991).

[5] Z. Jaroszewicz, Axicons: Design and Propagation Properties, Vol. 5 of Research and Development Treatise (SPIE Polish Chapter, Warsaw, (1997).

[6] A. N. Khilo, E. G. Katranji, and A. A. Ryzhevich, "Axicon-based Bessel resonator: analytical description and experiment," J. Opt. Soc. Am. A 18, 1986-1992 (2001).

[7] J. Rogel-Salazar, G.H.C. New, S. Chavez-Cerda, "Bessel-Gauss beam optical resonator," Opt. Communs. 190, 117 $122(2001)$.

[8] I. A. Litvin, A. Forbes "Bessel-Gauss resonator with internal amplitude filter," Opt. Communs. 281, 2385-2392 (2008).

[9] V.N.Belyi, N. S. Kazak, N.A. Khilo. "Properties of parametric frequency conversion with Bessel light beams," Optics Communs. 162, 169-176 (1999).

[10] N.A. Khilo, E.S. Petrova, A.A. Ryzhevich. "Transformation of the order of Bessel beams in uniaxial crystals," Quantum Electronics. 31, 85-89 (2001).

[11] V N Belyi, N S Kasak, N A Khilo, "Frequency conversion of Bessel light beams in nonlinear crystals," Quantum Electronics 30 (9), 753-766 (2000).

[12] Z. Bouchal, J. Wagner, M. Chlup, "Self-reconstruction of a distorted nondiffracting beam", Opt. Communs. 151, 207-212 (1998).

[13] N. Davidson, A. A. Friesem, and E. Hasman, "Holographic axilens: high resolution and long focal depth," Opt. Lett. 16, 523-525 (1991)

[14] Z. Jaroszewicz and J. Morales, "Lens axicons: systems composed of a diverging aberrated lens and a perfect converging lens," J. Opt. Soc. Am. A 15, 2383-2390 (1998).

[15] Christian Parigger, Y. Tang, D. H. Plemmons, and J. W. L. Lewis, "Spherical aberration effects in lens-axicon doublets: theoretical study," Appl. Opt. 36, 8214-8221 (1997).

[16] A.V. Goncharov, A. Burvall, and C. Dainty, "Systematic design of an anastigmatic lens axicon," Appl. Opt. 46, 6076-6080 (2007).

[17] T. Tanaka and S. Yamamoto, "Comparison of aberration between axicon and lens," Opt. Communs. 184, 113-118 (2000).

[18] T. Aruga, "Generation of long-range nondiffracting narrow light beams," Appl. Opt. 36, 3762-3768 (1997).

[19] T. Aruga, S. W. Li, S. Yoshikado, M. Takabe, and R. Li, "Nondiffracting Narrow Light Beam with Small Atmospheric Turbulence-Influenced Propagation," Appl. Opt. 38, 3152-3156 (1999).

[20] O. Brzobohatý, T. Cižmár, and P. Zemánek, "High quality quasi-Bessel beam generated by round-tip axicon," Opt. Express 16, 12688-12700 (2008).

[21] D. McGloin, K. Dholakia, "Bessel beams: diffraction in a new light," Contemporary Physics 46, 15-28 (2005). 